\title{
Alternative Masks for Nanolithography
}

\author{
D. Ingert
}

Laboratoire L.M.2.N., U.M.R. C.N.R.S. 7070, Université P. et M. Curie (Paris VI), B.P. 52, 4 Place Jussieu, F - 75231 Paris Cedex 05, France

\begin{abstract}
The use of masks coming from research field as different as colloids, polymers or nanomaterials is a recently emerging field. Recent advances in this area have developed a variety of practical routes which have a great potential to overcome or at least complete the high-cost lithographic techniques. This review focuses on three techniques that try to reduce to the nanometer range, the size of the mask. The major difference between these procedures is related to the type of mask used. The first technique is called colloidal lithography, the mask is a monodispersed-beads template. The second is the block copolymer lithography and the third technique is the nanocrystal lithography, the mask used is a nano-object. For these three parts, the synthetic routes, the improvements and the applications as well as the limitations will be presented.
\end{abstract}

\section{INTRODUCTION}

Nanotechnology research is led by the demand for ever smaller device features that are required to improve performance and decrease costs in microelectronics, communication and data storage. Lithographic methods are in the centre of this nanotechnology. Rapid replication of features below $50 \mathrm{~nm}$ is currently a significantly unsolved problem. Generating patterned surfaces at the nanoscale is beyond the limits of standard photolithography techniques. The wavelengths of photons limit the minimum feature sizes in the sub-100nm scale. Thus it appears that whatever technologies are used for fabrication in this region it does not represent simple engineering extensions of conventional photolithography. There exists an opportunity to introduce news ideas in replication of meso and nanostructures. Indeed, very few methods provide the ability to work in the sub-50-nm scale [1-3]. The well-established methods for the fabrication of nanometerscale structures [2], such as scanning probe, electron and ion beam lithography provide resolution and integration density required for the industrials demands but the level of throughput rate is far below the requirement. There is a need for alternative simple techniques in order to save processing time and costs $[4,5]$. This need has given rise to different kinds of approaches like, for example, nanoimprint lithography and soft lithography with the use of self-assembly monolayers (SAMs) [3,6,7]. However, for both the mold fabrication problems remain unsolved.

In this review we will focus on three techniques that try to reduce to the nanometer range, the size of the mask keeping a large surface coverage. The major difference between these procedures is related to the type of mask used while the standard techniques to pattern the substrate like metal deposition or ion-plasma etching are kept similar. The main requirements present in an efficient method for replication are flexibility in the engraved substrate, fidelity in the replication, low density of defects and large patterned surface. Because all of these characteristics are never present in a single

*Address correspondence to this author at the Laboratoire L.M.2.N., U.M.R. C.N.R.S. 7070, Université P. et M. Curie (Paris VI), B.P. 52, 4 Place Jussieu, F - 75231 Paris Cedex 05, France; E-mail: ingert@sri.jussieu.fr method, it is necessary to combine techniques. Thus we present these three techniques and their extensions combined with others. The first technique is called colloidal lithography, the mask is a monodispersed-spheres template (typically polystyrene beads with an average diameter of 200 $\mathrm{nm})$. The second is the block copolymer lithography, block copolymers are made of two chemically different chains bonded covalently. When the blocks are incompatible, they spontaneously self-assemble into micro domains, this leads, at equilibrium, to an ordered structure which is utilized as lithographic mask. The third technique is the nanocrystal lithography, the mask used is a nano-object.

\section{COLLOIDAL LITHOGRAPHY}

In 1981, Fischer and Zingsheim [8] proposed the use of a monolayer of spheres (about $300 \mathrm{~nm}$ ). One year later, Deckman and Dunsmuir [9] improved the technique in which spherical colloidal particles are used to define larger area lithographic masks. The deposition is either random or with ordered arrays over the entire surface of a macroscopic substrate [10]. The coating procedure developed to produce microcrystalline arrays is based on a spin-coating process, they also used a Langmuir-Blodgett-based technique [11]. It is necessary that the colloids wet the substrate surface. Then, the substrates are either pre-coated with a surfactant layer or a surfactant is added to the colloidal solution. On the tens of micrometers scale, points defects and dislocations are present [9-11], this is related to the formation of hexagonally close-packed arrays which is a two-step mechanism with nucleation and growth [12]. Then in the 90s, Van Duyne explored the versatility of the technique, which was called NanoSphere Lithography [13-14]. His group changed either the substrate (semiconductor, metal or insulator) or the deposition material (metal, organic semiconductor and insulator). They use two ways to deposit the nanospheres: spin coating or hand (drop) coating. The technique was then improved to control not only the shape of the ending particles but also the shape of the periodic particle array [15]. To do this, colloids having different diameters organized in a single-layer or a double-layer as a mask are used. With single-layer nanosphere arrays, the ending structure is made of triangular particles organized in a honeycomb structure. A schematic 


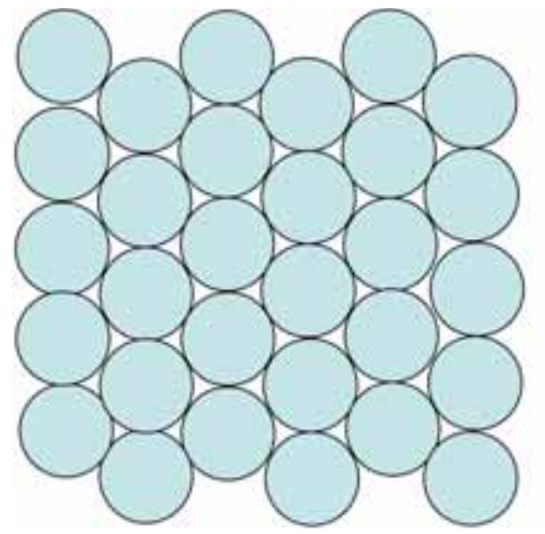

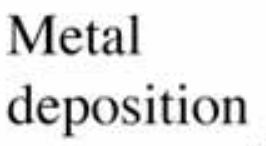

and beads removing

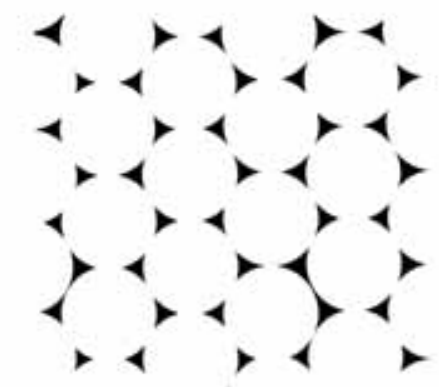

Fig. (1). Schematic representation of nanosphere lithography.

representation is given in Fig. (1). Indeed, the deposited material passes through the 3 -fold triangular-shaped interstices in the mask. The double-layer nanosphere mask is prepared using higher colloid solution concentrations. In this case, the evaporated material passes through the 6-fold hexagonal-shaped interstices in the mask. This leads to elliptical-shape particles organized in a hexagonal network. More recently, this group described a novel approach to tune the shape of the final particles. This approach, called angleresolved nanosphere lithography [16], is a variant of the standard nanosphere lithography. This is accomplished by controlling the angle between the normal to the surface of the sample assembly and the propagation vector of the material deposition beam [16-17]. This makes it possible to reduce the in-plane particle dimensions.

Another very simple method to obtain a colloid monolayer is to deposit a droplet of a colloid solution on a glass plate and control the evaporation time and the temperature [18-19]. Leiderer et al. developed a method to use almost any substrate [20-22]. The entire monolayer is transferred from the glass substrate onto a water surface. From there, it is transferred to the desired substrate by contacting it from above with a horizontally held substrate.

\section{Combining with Other Techniques}

Nanosphere lithography has two major disadvantages. First, the geometry of the mask of colloidal particles is limited since it is created by self-organization: for a single layer the only structure is hexagonal close packed. Second, the size and spacing of the mask holes are couple, and thus these properties cannot be independently controlled. As we have present, conventional self-assembly methods could be applied to produce 2D and 3D ordered hexagonal close-packed (hcp) arrays of colloidal spheres. By combining with etching techniques or using charged spheres, non close-packed arrays with controllable spacing between spheres could be created even if it is still difficult to obtain new lattice structures other than hexagonal arrays.

As described above, Deckman et al. [9] used random arrays of colloidal particles to texture surfaces. Random spheres arrangements are produced because polyballs adhere to the substrate after diffusing through the aqueous colloidal suspension close to the substrate surface. Indeed, when an electrostatic attraction is set up between a substrate and an aqueous suspension of charged polyballs, a randomly- arranged monolayer-thick coating can be obtained. Charged spheres adsorb randomly onto an oppositely-charged surface, and are arranged through electrostatic interactions. The randomness of the initial interactions of the colloids produces uniformity over large surface areas. This can be an advantage compared to the hexagonally close-packed arrays where dislocations and points defects are common. In this case, the substrate coverage is controlled and, consequently, the average distance between colloids [23-24]. This is obtained by changing the colloid concentration or by adding salt to the colloidal solution. With this deposition process, combined with a heat treatment leading to a shape modification of the beads, gold nanodisks have been formed [25].

Yan et al. [26] report the utilization of the solventswelling and mechanical deformation behaviors of polydimethylsiloxane elastomer (PDMS) to adjust the lattice structures of 2D arrays spheres (Fig. 2). Silica spheres were assembled into hcp arrays on a silicon wafer. By using the liftup soft lithography, a single layer of hcp spheres was transferred to the surface of PDMS film, which was subsequently stretched or swollen with solvent solution to transform the hcp array into non close-packed one. This PDMS film was then transferred to the substrate by using micro-contact printing.

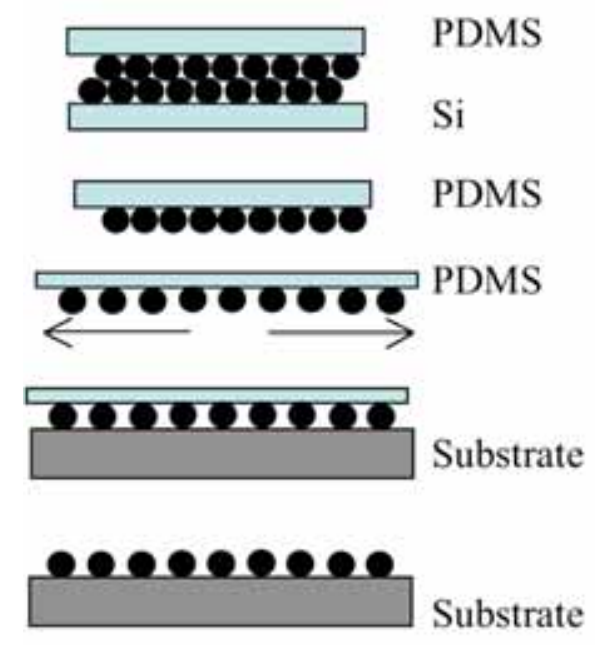

Fig. (2). Schematic representation of the procedure for the fabrication of 2D non compact arrays of spheres with tunable lattice structure 
Vossen et al. [27] present a combination of two methods to resolve these limitations. They create colloidal mask with arbitrary geometry using optical tweezers and they tune the shape mask using ion irradiation which causes the colloids to expand in the in plane direction. These different techniques allow to overcome the limitations of the colloidal lithography with the modulation of the size and the distance between objects done independently in one side, and with the tunnability in the pattern shape in the other other side, but they are more time consumming compared to the basical technique.

The colloidal lithography leading to nanostructured surfaces with defined roughness and periodicity finds several applications, for example, in optical studies, catalysis, magnetism or biological devices. Here, we will just mention a few of these. Van Duyne et al. [28-29] made in-depth studies of the optical properties, the surface plasmon resonance, of periodic arrays of silver particles. Indeed, by nanosphere lithography, as described previously, the shape, size and particle spacings are tuned. This was extended to surfaceenhanced Raman spectroscopy (SERS) [30]. Another application is based on the understanding of catalytic processes. In the fabrication of a model catalyst, the kinetic parameters such as particle size and shape, interparticle distances or the influence of the substrate [31-32] is controlled. To conclude this list of the applications of colloidal lithography, one of the very promising fields is biology. It has been demonstrated that arrays of metal particles can be used as nanosensors by binding biological molecules to them [33-35]. Another use could be the fabrication of implants where the nanostructuration plays a very efficient role [36-39].

\section{BLOCK COPOL YMERS}

Though block copolymers have been largely studied for several decades, it is only in the last few years that they have been used for nanotechnology [40]. Block copolymers are made of two chemically different chains bonded covalently. When the blocks are incompatible, they spontaneously selfassemble into micro domains, this leads, at equilibrium, to an ordered structure. Owing to their mutual repulsion, dissimilar blocks tend to segregate into different domains, the spatial extent of the domains being limited by the constraint imposed by the chemical connectivity of the blocks. Parameters like the chemical composition of the different blocks, the length or the molar mass control the micro-phases morphology, their size or periodicity. This high adjustability enables the topography of the block copolymers pattern to be of interest for nanolithography masks [41-43].

Indeed, one block relative to the other block of the copolymer has chemical or physical differences. This involves a selective process, e.g., the etching rate is different between the two blocks, leading to the formation of a template of either a porous network or arrays of dots [44-46]. Various methods were developed to enhance the etch selectivity. A common approach feature is to have one of the block phases containing suitable inorganic components [47-52]. This makes it possible to achieve etching contrast. This template is then used for pattern transfer, either by direct replication into the substrate through etching or as a growth matrix. Mansky et al. [41-42] first demonstrated that monolayer films of diblock copolymer microdomains could potentially be used as masks for nanolithography, on the scale of a few tens of nanometers. Park et al. [44] obtained dense periodic arrays of holes and dots by spin-coating diblock copolymer thin films. The ending holes, $20 \mathrm{~nm}$ across hexagonallyordered with $40-\mathrm{nm}$ spacing with polygrain structure were obtained, yielding a pattern with approximately $10^{11}$ holes $/ \mathrm{cm}^{2}$.

The main limitation in using block copolymers as lithographic masks is that it is hard to control the order on the global scale even if locally the self-assembled structure is very precise. Indeed, self-assembly of block copolymers leads to polygrain structures with defects such as grain boundaries and/or dislocations. Several techniques are developed to induce long-range order of the microdomains of block copolymers. They are based on achieving anisotropic properties of the polymer. The methods currently used in the polymer field are applying mechanical flow fields, temperature gradients, electric fields or solvent control [40].

\section{Combining with Others Lithographic Techniques}

Another way to provide better control of block copolymer microdomains is to use other lithographic techniques in order to pre-pattern the substrate. For example, selfassembly monolayers (SAM's) allow chemically modifying the surface pattern [53-54]. This leads to a higher homogeneity of the interactions between the block copolymer and the substrate [55]. The substrates can also be patterned topographically. Micropatterning a substrate is done with a PDSM pattern, which can be generated by soft lithography and used as a mold for block copolymers [56-57]. Micropatterning of a single layer of nanoparticles has been done by combining a self-assembly of diblock copolymer micelles with conventional and soft lithography [58]. On a photoresist micropattern fabricated by conventional photolithography, a single layer of diblock copolymer micelles containing precursors of nanoparticles was spin coated. Oxide nanoparticles were synthesized by oxygen plasma treatment on the single layer, and then unnecessary photoresists were lifted off. Thus micropatterns of nanoparticles were successfully produced on the substrate. Second, soft lithography of the microcontact printing technique was combined with the process of diblock copolymer micelles to produce micropatterns of nanoparticles. A single layered film of diblock copolymer micelles was spin coated onto a microcontact printing stamp, and then was transferred on the substrate by stamping. Formation of nanoparticles in the micropattern was carried out by the procedure of plasma treatment.

Standard lithography is used to fabricate grating substrates with, for example, microscale periodicity and various heights. Ordered arrays are, in this way, formed by spin casting a block copolymer over surfaces patterned with shallow grooves [59-60]. An illustration of this is in Fig. (3), where the bands, patterned by interference lithography, control the nanostructure location. By a similar process, dots that are organized in rings or stars, are also achieved by prepatterning generated by photo or e-beam lithography [51]. This leads to a large variety of rather complex structures on the mesoscale with dot- arrays inside on the nanoscale.

Block copolymer lithography has led, by combination with standard semiconductor lithography techniques, to, for example, the fabrication of semiconductor capacitors. An- 
other field, where this lithography is involved, is the production of magnetic structures [50,52]. For example, cobalt nanodots arrays have been prepared; they are small enough to be a single magnetic domain. Jung et al. [61] have performed protein nanoarrays by using block copolymer lithography in order to immobilize proteins at predefined positions. The regular pattern of biofunctionalized templates provides a useful tool for elucidating recognition events with the result of enhanced biosensor performance through the use of these novel protein-immobilization strategies. The biopattern themselves are involved in masking [62,63].

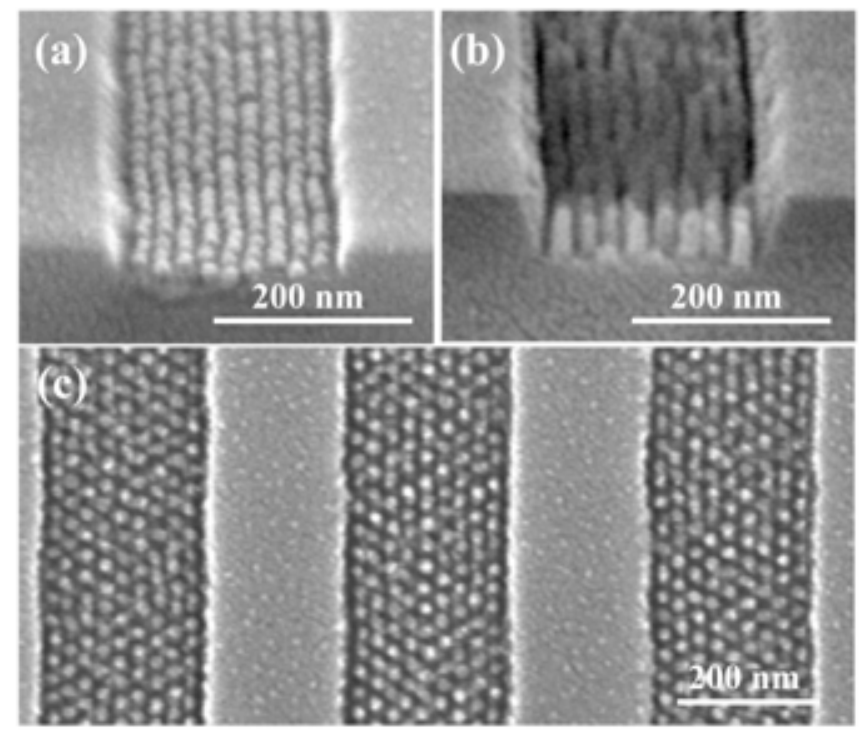

Fig. (3). Pattern transfer from an ordered PS/PFS 50/12 polymer to form an array of ordered silica nanostructures. (a) Side view of the PFS pattern in 240-nm wide grooves. (b) Side view and (c) plan view of the pattern after it has been transferred into an underlying silica layer bye reactive ion etching. The silica posts in (c) have the same spatial organization as the originating PFS features and aspect ratios exceeding 3. Reprinted with permission from J. Y. Cheng, C. A. Ross, E. L. Thomas, Henry I. Smith, and G. J. Vancso, Applied Physics Letters, 81, 3657 (2002). Copyright 2002, American Institute of Physics.

\section{NANOCRYSTALS LITHOGRAPHY}

A new type of mask seems to emerge in the nanolithographic field, they are no more composed by soft matter like PS beads or copolymers, but they come from the material research and are nanocrystals. They open a new opportunity to overcome the actual limitation in the lithography. Indeed, they are nanoobjects and offer a great versatility in terms of shape, nature of material and organization.

\section{Mesoscopic Scale}

Organization of nanocrystals or individual nanocrystals are used as lithographic mask, this allows to get different geometries of pattern depending on the shape of the organization at the mesocopic scale or on the shape of the nanocrystal itself [64]. The selective etching process is based on the difference of etching rate between the nanomaterial and the engraved substrate. Different substrates are patterned with this technique.
To etch silica substrate, two-step Reactive Ion Etching (RIE) is performed. The substrate has to be first spin coated with a polymer film (PMMA). Indeed, $\mathrm{Si} / \mathrm{SiO}_{2}$ is commonly etched with SF6 plasma but this plasma attacks also the nanocrystals mask. This "multilayer" substrate ( $\left.\mathrm{SiO}_{2} / \mathrm{PMMA}\right)$ makes it possible to increase the etching depth. Ferrite nanocrystals [65-66] are deposited onto the multilayer substrate. Different nanocrystals deposition methods are used depending on the geometry of the desired mask. Rings or lines made of nanocrystals can be formed. Using anticapillarity tweezers to maintain the substrate during the deposition of a droplet of the nanocrystals solution leads to the formation of rings due to Marangoni instabilities [67,68] (Fig. 4B). If the substrate is immersed in a nanocrystals solution and a magnetic field is applied during the evaporation process, lines made of nanocrystals are formed [69,70] (Fig. 4A). A first RIE $\left(\mathrm{O}_{2}\right)$ etches the polymer leading to the formation of a pre-mask. This makes it possible to increase the depth of the mask and thus reach the silica substrate. The second RIE step involves a $\mathrm{SF}_{6}$ plasma which will etch this substrate (Fig. 4C). The ring geometry is kept before and after etching (Fig. 5A,B). Analyses (EDX and XDR) prove that the transferred ring pattern is made of $\mathrm{SiO}_{2}$. Hence, mesostructures are reproduced in a given substrate through a nanocrystals mesoscopic organization used as a lithographic mask [64]. This is the first example where the use of nanocrystals as masks is demonstrated. Even if the diameter remains equivalent, a loss in the ring profile resolution is observed compared to that obtained before etching. This is explained by the inhomogeneity in the thickness of the pattern mask. In fact, a sinusoidal thickness profile of the mask induces the increase in the width of the etching replica [71]. Thus, this technique loses in resolution if the mask height is not homogeneous. Line geometry has the advantage of the same average height. After etching, the dimension and the resolution of the lines are retained (Fig. 5C,D). The precision of the lines remains equivalent before and after etching. This indicates that the resolution transfer improves with increasing the regularity of the mask relief. As with rings, lines made of ferrite nanocrystals play a very efficient role in a transferring mask. This technique makes it possible to pattern a large surface area in a very short time. In this range (hundred nanometers), core shell nanowires $\left(\mathrm{Si} / \mathrm{SiO}_{2}\right)$ with controlled diameter and shell dimensions were used as mask for etching and deposition [73]. Surfactant stabilized nanowires are compressed on a Langmuir-Blodgett trough to produce aligned nanowires with an interwire distance controlled by compression process and by the core diameter and shell thickness that are control independently. Indeed, below a pitch of $100-200 \mathrm{~nm}$, the compression leads to increasing aggregation due to strong internanowire attractive forces which enables control the distance between nanowires at the nanometer scale. The aligned nanowires are then horizontally transferred onto hydrophobic substrates to form uniformly ordered parallel arrays. Selective anisotropic etching is used to transfer the line-pattern to the underlying substrate surfaces. In addition, other materials, such as metals, can be deposited using the aligned nanowires as shadow mask to create arrays of wires. Finally, the nanowire masks are removed by isotropic wet etching and sonication to expose the etched or deposited parallel line features. 


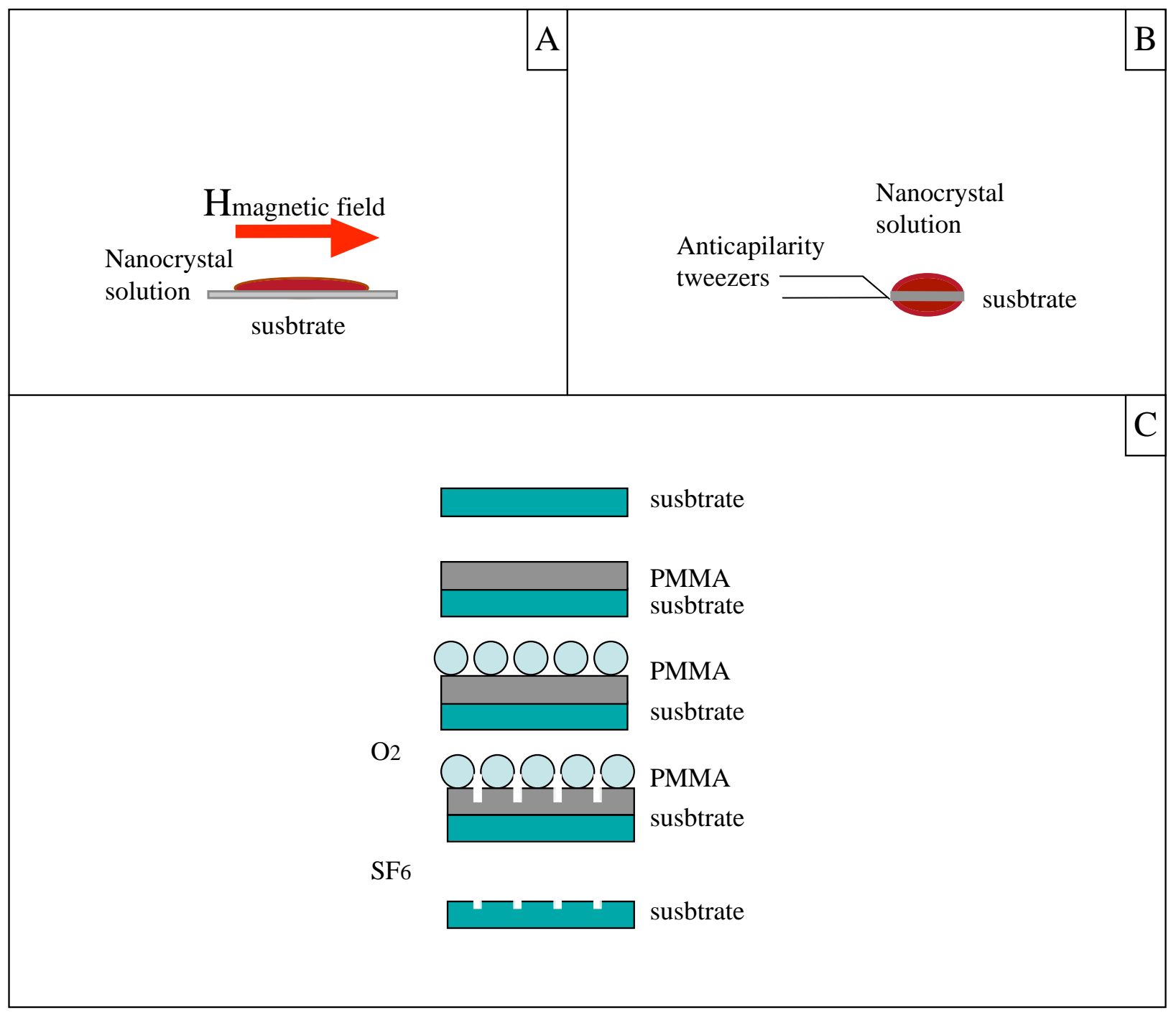

Fig. (4). Schematic illustration of the deposition methods to obtain lines (A) and rings (B) and of the general etchig process.

\section{Nanometric Scale}

After employing mesostructures, an individual nanocrystal is used as a lithographic mask to reach a resolution of about ten nanometers. The mask is hematite needles with a diameter of $40 \mathrm{~nm}$ and length of $280 \mathrm{~nm}$. The transferred individual needles are very well defined. This means that it is possible to use nanocrystals with a smaller dimension of about ten nanometers as lithographic masks. This is, to our knowledge, the first time that an individual nanocrystal is used as a mask [72]. Nevertheless, it must be noted that although this method is good enough to pattern dimensions of about ten nanometers, it is not satisfactory for lower dimensions (a few nanometers). When needles are packed in 2D, which means with height homogeneity and separated by few nanometers, in the transferred pattern, the outline is no more discernable. A spacing between objects less than $10 \mathrm{~nm}$ can be deduced. This means the average distance between nanocrystals is a crucial parameter for the transfer. In order to prove that this technique really achieves spacings smaller than $10 \mathrm{~nm}$, FEG-SEM patterns are obtained. Fig. (6A) shows clearly that the isolated needle is well separated from the "V" needle. The distance between them is $9.3 \mathrm{~nm}$ (Fig.
6B) and clearly confirms that with the technique described here it is possible to reach a spacing smaller than $10 \mathrm{~nm}$.

Silicon nanocrystals are fabricated using platinum nanomask [74]. The $\mathrm{SiOx}$, amorphous $\mathrm{Si}$ and $\mathrm{SiOx}$ thin films are successively deposited on a $\mathrm{Si}$ wafer by using radio frequency magnetron sputtering. Then, nanosized Pt islands are deposited uniformly on the top of the as prepared substrate by using direct current magnetron sputtering method. The $\mathrm{Pt}$ islands play the role of etching masks for RIE process. Finally, after the deposition of $\mathrm{SiOx}$ gate oxide layer and the removal of Pt islands using aqua regia, the $\mathrm{Si}$ nanocrystals embedded in the $\mathrm{SiO}_{2}$ are recristallized during thermal annealing.

\section{Overcoming the Mask Limitation}

Rogers and al. have used single walled carbon nanotubes (SWNTs) as template of the PDMS stamp in soft nanoimprint lithography [75-76]. They have shown that SWNTs can serve as templates for performing polymer imprint lithography with feature sizes as small as $2 \mathrm{~nm}$. They showed that for the molecular scale, the limitation is not either induced by the mask but by, in this case, the density of cross-links of the "engraved" polymer surface. 


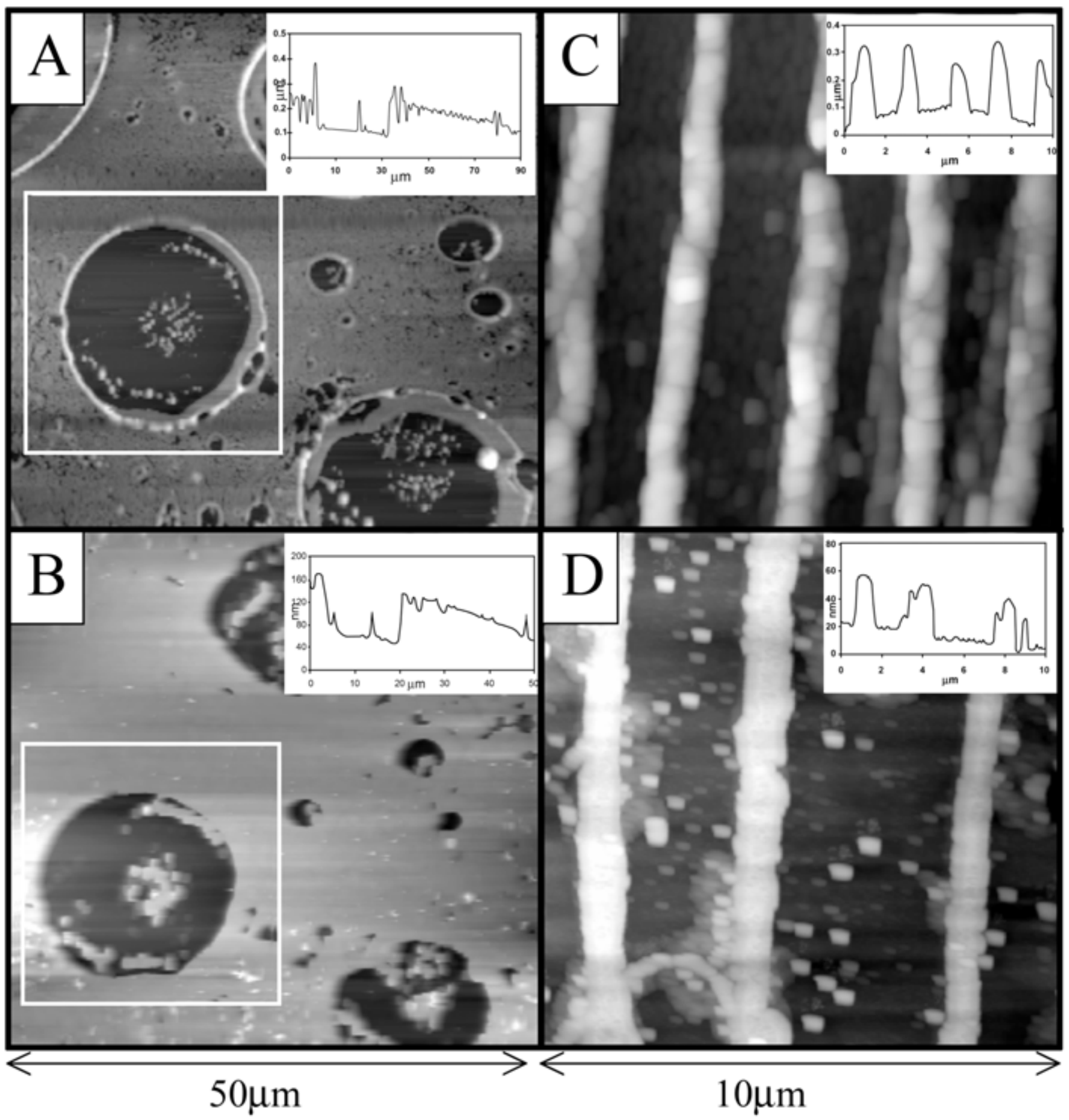

Fig. (5). (A) and (B) AFM images of a ring made of 10-nm maghemite obtained before and after etching process, respectively, with the cross sections in insert. (C) and (D) AFM images of lines before and after etching, respectively, with the cross sections in insert.

\section{Summary and Future Outlook}

We have presented three techniques that can be called "colloidal lithography" and have discussed the general features and the state of the art. Spontaneous formation of wellordered "colloids" arrays provides lithographic masks for creating useful patterns. However, it is necessary to solve the problems of defect formation during self-assembly. To this end, one of the solution is to confine the area or treat the surfaces using others lithographic techniques. The combination with others methods also permits to get different geometries of arrays. These new patterns find applications in field as different as magnetism, electronics or biology. The noted applications constitute only some of the potential. Nanosphere Lithography can pattern a rather large surface area with defined ending particle shapes. The smallest spacing that has been attained with usual nanosphere lithography is about $50 \mathrm{~nm}$. The very recent development in this field 
shows the fabrication of very robust structures that are employed in chemical and biological sensing [77]. The nanoparticles arrays on flat surfaces are robust and stable in organic solvents. The application particularly in biology need device with the same characteristic (strong adhesion of the nanostructure under a liquid flow) but in aqueous solution as is any biological medium. The system used is to anchor nanoparticles into the substrate. The mechanical strength is obtained but it is attended by a decrease in the uniformity of the structure and the etching process still need improvement. With the block copolymer lithography, the dot/cylinder shape is most commonly obtained. The main limitation is to get well-resolved periodic arrays over a large area; the developments of this technique are mainly based on combining it with patterns obtained with standard lithography methods. Block copolymer nanofabrication can provide large area periodic functional structures or objects with feature size of the order of tens of nanometers. However, in many applications, such as multifunctional on-chip bioseparations, simple periodic structure is insufficient and spatial control of the microdomains is necessary [78]. Further research in this field will doubtless help stimulate development of the next generation of devices. In Nanocrystal Lithography, a resolution down to $10 \mathrm{~nm}$ can be reached which starts to be competitive with electron lithography. To reach the few-nanometer scale, individual nanocrystals or 2D organizations could be suitable masks but the patterning technique has to be changed.

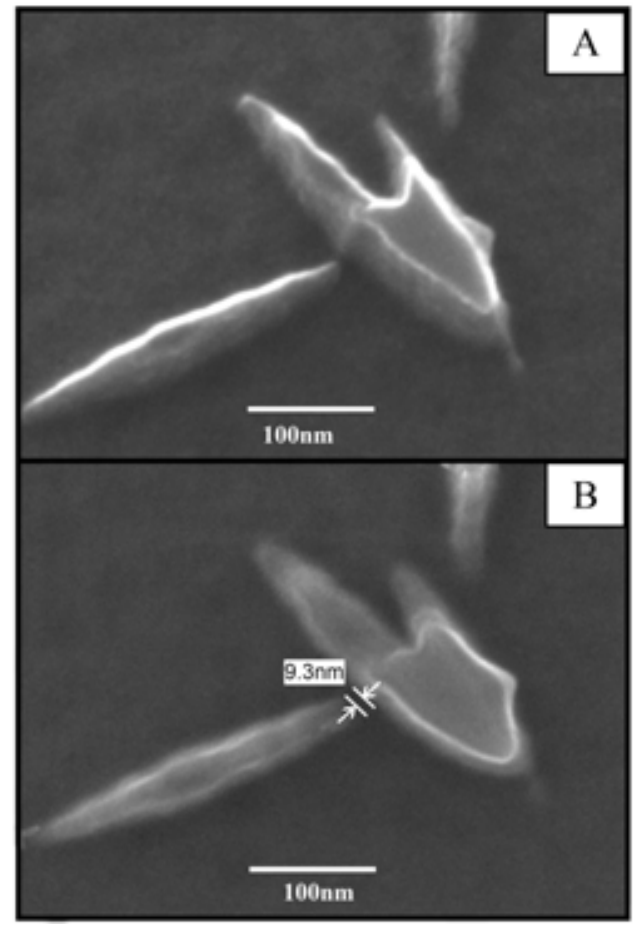

Fig. (6). FEG-SEM images of transferred needles with the smallest spacing (A) tilted $10^{\circ},(\mathbf{B})$ non-tilted.

\section{REFERENCES}

[1] Azzaroni, O.; Fonticelli, M.; Schilardi, P.L.; Benitez, G.; Caretti, I.; Albella, J.M.; Gago, R.; Vasquez, L.; Salvarezza, R.C. Nanotechnology, $2004,15, \mathrm{~S} 197$.

[2] Martin, J.I.; Nogués, J.; Liu, K.; Vicent, J.L.; Schuller, I.K. JMM, 2003, 256, 449.

[3] Xia, Y.; Rogers, J.A.; Paul, K.E., Whitesides, G.M. Chem. Rev., 1999, 99, 1823.
[4] Smith, H.I. Physica E, 2001, 11, 104.

[5] Michel, B.; Bernard, A.; Bietsch, A.; Delamrche, E.; Geissler, M.; Juncker, D.; Kind, H.; Renault, P.; Rothuizen, H.; Schmid, H.; Schmidt-Winkel, P.; Stutz, R.; Wolf, H. IBM J. Res. Dev., 2001, 45, 697

[6] Qin, D.; Xia, Y.; Xu, B.; Yang, H.; Zhu, C.; Whitesides, G.M. Adv. Mat., 1999, 11, 1433.

[7] Hammond, P.T. Adv. Mater., 2004, 16, 1271.

[8] Fischer, U.C.; Zingsheim, H.P. J. Vac. Sci. Technol., 1981, 19, 881

[9] Deckman, H.W.; Dunsmuir, J.H. Appl. Phys. Lett., 1982, 41, 377.

[10] Deckman, H.W.; Dunsmuir, J.H. J. Vac. Sci. Technol. B, 1983, 4, 1109.

[11] Deckman, H.W.; Dunsmuir. J.H. Garoff, S.; McHenry, J.A.; Pfeiffer, D.G. J. Vac. Sci. Technol. B, 1988, 6, 333 .

[12] Denkov, N.D.; Velev, O.D.; Kralchevsky, P.A.; Ivanov, I.B.; Yoshimura, H.; Nagayama, K. Langmuir, 1992, 8, 3183.

[13] Hulteen, J.C.; Van Duyne, R.P. J. Vac. Sci. Technol. A, 1995, 13, 1553.

[14] Hulteen, J.C.; Treichel, D.A.; Smith, M.T.; Duval, M.L.; Jensen, T.R.; Van Duyne, R.P.; J. Phys. Chem. B, 1999, 103, 3854.

[15] Haynes, C.L.; Van Duyne, R.P. J. Phys. Chem. B, 2001, 105, 5599.

[16] Haynes, C.L.; McFarland, A.D.; Smith, M.T.; Hulteen, J.C.; Van Duyne, R.P. J. Phys.Chem. B, 2002, 106, 1898.

[17] Kosiorek, A.; Kandulsky, W.; Chudzinski, P.; Kempa, K.; Giersig, M. Nanolett., 2004, 4, 1359.

[18] Micheletto, R.; Fukuda, H.; Ohtsu, M. Langmuir, 1995, 11, 3333.

[19] Kralchevsky, P.A.; Nagayama, K. Langmuir, 1994, 10, 23.

[20] Burmeister, F.; Schäfle, C.; Mattes, T.; Böhmisch, M.; Boneberg, J.; Leiderer, P. Langmuir. 1997, 13, 2983.

[21] Boneberg, J.; Burmeister, F.; Schäfle, C.; Leiderer, P.; Reim, D.; Fery, A.; Herminghaus, S. Langmuir, 1997, 13, 7080.

[22] Burmeister, F.; Schäfle, C.; Keihofer, B.; Bechinger, C.; Boneberg, J.; Leiderer, P. Adv. Mat., 1998, 10, 495.

[23] Hanarp, P.; Sutherland, D.S.; Gold, J.; Kasemo, B. Coll. Surf. A, 2003, 214, 23.

[24] Denis, F.A.; Hanarp, P.; Sutherland, D.S.; Dufrêne, Y.F. Nanolett., 2002, 2, 1419.

[25] Hanarp, P.; Käll, M.; Sutherland, D.S. J. Phys. Chem. B, 2003, 107, 5768.

[26] Yan, X.; Yao, J.; Lu, G.; Li , X.; Zhang, J.; Han, K.; Yang, B. JACS, 2005, 127, 7688.

[27] Vossen, D.L.J.; Fific, D.; Penninkhof, J.; van Dillen, T.; Polman, A.; van Blaaderen, A. Nanolett., 2005, 5, 1175.

[28] Jensen, T.R.; Schatz, G.C.; Van Duyne, R.P. J. Phys. Chem. B, $1999103,2394$.

[29] Jensen, T.R.; Duval, M.L.; Kelly, K.L.; Lazarides, A.A.; Shatz, G.C.; Van Duyne, R.P. J. Phys. Chem. B, 1999, 103, 9846.

[30] Haynes, C.L.; Van Duyne, R.P. J. Phys. Chem. B, 2003, 107, 7426

[31] Kasemo, B. Top. Catal., 2003, 13, 43.

[32] Werdenius, C.; Österlund, L.; Kasemo, B. Langmuir, 2003, 19 458.

[33] Haes, A.J.; Van Duyne, R.P. J. Am. Chem. Soc., 2002, 124, 10596.

[34] Haes, A.J.; Van Duyne, R.P. Exp. Rev. Mol. Diagn., 2004, 4, 527.

[35] Haes, A.J.; Hall, W.P.; Chang, L.; Klein, W.L.; Van Duyne, R.P. Nanolett., 2004, 4, 1029.

[36] Denis, F.A.; Hanarp, P.; Sutherland, D.S.; Gold, J.; Mustin, C.; Rouxhet, P.G.; Dufrêne, Y.F. Langmuir, 2002, 18, 819.

[37] Spupinski, M.; Sanz, R.; Jensen, J. Nucl. Instr. Meth. Phys. Res. B, 2007, 257, 777.

[38] Ueda, K.; Utsumi, M. Polyhedron, 2007, 26, 2381.

[39] Wang, B.; Zhao, W.; Chen, A.; Chua, S.J. J. Cryst. Growth, 2006 288, 200.

[40] Park, C.; Yoon, J.; Thomas, E.L. Polymer, 2003, 44, 6725.

[41] Mansky, P.; Chaikin, P.; Thomas, E. J. Mat. Sci., 1995, $30,1987$.

[42] Mansky, P.; Huang, E.; Liu, Y.; Russel, T.P.; Hawker, C. Science, 1997, 275, 1458

[43] Huang, E.; Rockford, L.; Russel, T.P.; Hawker, C.J. Nature, 1998 395, 757.

[44] Park, M.; Harrison, C.; Chaikin, P.M.; Register, R.A.; Adamson, D.H. Science, 1997, 276, 1401.

[45] Li, R.R.; Dapkus, P.D.; Thompson, M.E.; Jeong, W.G.; Harrison, C.; Chaikin, P.M.; Register, R.A.; Adamson, D.H. Appl. Phys. Lett., 2000, 76, 1689.

[46] Turn-Albrecht, T.; Schotter, J.; Kästle, G.A.; Emley, N.; Shibauchi, T.; Krusin-Elbaum, L.; Guarini, K.; Black, C.T.; Tuominen, M.T.; Russell, T.P. Science, 2000, 290, 2126. 
[47] Spatz, J.P.; Eibeck, P.; Mössmer, S.; Möller, M.; Herzog, T.; Ziemann, P. Adv. Mat., 1998, 10, 849 .

[48] Koslowski, B.; Strobel, S.; Herzog, T.; Heinz, B.; Boyen, H.G.; Notz, R.; Ziemann, P.; Spatz, J.P.; Möller, M. J. Appl. Phys., 2000, 87,7533 .

[49] Lammertink, R.G.H.; Hempenius, M.A.; van den Enk, J.E.; Chan, V.Z.H.; Thomas, E.L.; Vancso, G.J. Adv. Mat., 2000, 12, 98.

[50] Cheng, J.Y.; Ross, C.A.; Chan, V.Z.H.; Thomas, E.L.; Lammertink, R.G.H.; Vancso, G.J. Adv. Mat., 2001, 13, 1174.

[51] Glass, R.; Möller, M.; Spatz, J.P. Nanotechnology, 2003, 14, 1153.

[52] Choi, D.G.; Jeong, J.R.; Kwon, K.Y.; Jung, H.T.; Shin, S.C.; Yang, S.M. Nanotechnology, 2004, 15, 970.

[53] Kumar, A.; Whitesides, G.M. Appl. Phys. Lett., 2002, 1993, 63.

[54] Xia, Y.; Whitesides, G.M. Ann. Rev. Mater. Sci., 1998, 28, 153.

[55] Heier, J.; Genzer, J.; Kramer, E.J.; Bates, F.S.; Krausch, G. J. Chem. Phys., 1999, 111, 11101.

[56] Deng, T.; Ha, Y.H.; Cheng, J.Y.; Ross, C.A.; Thomas, E.L. Langmuir, 2002, 18, 6719 .

[57] Choi, D.G.; Yu, H.K.; Yang, S.M. Mat. Sci. Eng. C, 2004, 24, 213.

[58] Yun, S.H.; Sohn, B.H.; Jung, J.C.; Zin, W.C.; Ree, M.; Park, J.W. Nanotechnology, 2006, 17, 450.

[59] Park, C.; Cheng, J.Y.; Fasolka, M.J.; Mayes, A.M.; Ross, C.A.; Ross, E.L.; Thomas, E.L.; DeRosa, C. Appl. Phys. Lett., 2001, 79, 848 .

[60] Cheng, J.Y.; Ross, C.A.; Thomas, E.L.; Smith, H.I.; Vancso, G.J. Appl. Phys. Lett., 2002, 81, 3657.

[61] Jung, J.M.; Kwon, K.Y.; Ha, T.H.; Chang, B.H.; Jung, H.T. Small, 2006, 2, 1010.
Panhorts, M.; Brückl, H.; Kiefer, B.; Reiss, G.; Santarius, U.; Guckenberger, R. 2001, J. Vac. Sci. Technol. B, 19, 722.

Blawas, A.S.; Reichert, W.M. Biomaterials, 1998, 19, 595. Ingert, D.; Pileni, M.P. J. Phys.Chem. B, 2003, 107, 9617.

Ngo, A.T.; Pileni, M.P. Adv. Mat., 2000, 12, 276.

Ngo, A.T.; Pileni, M.P. J. Phys. Chem. B, 2001, 105, 53.

Maillard, M.; Motte, L.; Pileni, M.P. Adv. Mat., 2001,13, 200.

Maillard, M.; Motte, L.; Ngo, A.T.; Pileni, M.P. J. Phys. Chem. B, 2000, 104, 11871.

Lalatonne, Y.; Motte, L.; Richardi, J.; Pileni, M.P. Phys. Rev. E, 2005, 71, 011404

Pileni, M.P.; Lalatonne, Y.; Ingert, D.; Lisiecki, I.; Courty, A Faraday Discussions, 2004, 125, 251.

Huang, C.J.; Zhu, X.P.; Li, C.; Zuo, Y.H.; Cheng, B.W.; Li, D.Z Luo, L.P.; Yu, J.Z, Wang, Q.M. J. Cryst. Growth, 2002, 236, 141. Ingert, D.; Pileni, M.P. 2007, Submitted.

Whang, D.; Jin, S.; Lieber, C.M.; Nanolett., 2003, 3, 951

Lee, D.S.; Shim, Y.S.; Cho, H.Y.; Kang, T.W.; Kim, D.Y.; Lee, Y.H.; Wang, K.L. Thin Solid Films, 2004, 379, 451.

Hua, F.; Sun, Y.; Gaur, A.; Meitl, M.A.; Bilhaut, L.; Rotkina, L.; Wang, J.; Geil, P.; Shim, M.; Rogers, J.A. Nanolett., 2004, 4, 2467. Feng, H.; Gaur, A.; Yugang, S.; Word, M.; Niu, J.O.; Adesida, I.; Shim, M.; Shim, A.; Rogers, J.A.; IEEE Trans. Nanotech., 2006, 5, 301.

Hicks, E.M.; Lyandres, O.; Hall, W.P.; Zou, S.; Glucksberg, M.R.; Van Duyne, R.P.; J. Phys.Chem. C, 2007, 111, 4116.

Bratton, D.; Yang, D.; Dai, J.; Ober, C.K. Polym. Adv.Technol., 2006, 17,94 . 